\title{
Perfil de partos e nascimentos na baixada Maranhense
}

\author{
Profile of births in downtown Maranhense \\ Perfil de nacimientos en el centro de Maranhense
}

Deyvison de Lacerda Lopes $^{1 *}$, Sueli de Souza Costa ${ }^{1}$, Izolda Souza Costa ${ }^{2}$, Nilson de Jesus Pereira Batalha Júnior², Paola Trindade Garcia².

\section{RESUMO}

Objetivo: Caracterizar e analisar o perfil epidemiológico de nascimentos na Baixada Maranhense, no período 2011-2017. Métodos: Estudo epidemiológico descritivo, retrospectivo, de abordagem quantitativa, utilizando os dados das Declarações dos Nascidos Vivos (DNV), disponíveis no sítio do SINASC na internet. Resultados: Os números totais de nascidos vivos foi de 66.972 , sendo $51,5 \%$ do sexo masculino; $91,8 \%$ ocorreram em ambiente hospitalar; mães com idade entre 10 a 24 anos $(61,76 \%)$; maioria de cor parda $(82,65 \%)$; solteiras $(46,47 \%) ; 70,71 \%$ dos nascimentos foram a termo; houve predomínio de partos vaginais $(68,98 \%)$, com $30,34 \%$ de cesarianas; $65,36 \%$ das mães realizaram menos que sete consultas pré-natal; $7,49 \%$ dos nascituros com baixo peso ao nascer; houve correlação positiva entre partos cesáreos e: faixas etária 25 a 35 anos $(r=0,974 ; p<0,001)$; mães com mais de 35 anos $(r=0,836 ; p=0,019)$; escolaridade entre 8 a 11 anos $(r=0,936 ; p=0,012)$; gestações múltiplas $(r=0,652 ; p=0,024)$; baixo peso ao nascer $(r=0,635$; $p=0,048)$. Conclusão: Os dados do SINASC permitiram caracterizar a população materno-infantil nesta microrregião, apesar do grande número de informações ignoradas, reforçando a necessidade de capacitação e maior atenção ao preenchimento da DNV, a fim de gerar informações capazes de contribuir no planejamento das ações voltadas para este grupo.

Palavras-chave: Nascidos vivos, Perfil epidemiológico, Saúde materna, Saúde pública.

\begin{abstract}
Objective: To characterize and analyze the epidemiological profile of births in Baixada Maranhense, in the period 2011-2017. Methods: Descriptive, retrospective, epidemiological study with a quantitative approach, using data from Declarations of Live Births (DNV), available on the SINASC website. Results: The total number of live births was 66,972 , with $51.5 \%$ being male; $91.8 \%$ occurred in a hospital environment; mothers aged 10 to 24 years $(61.76 \%)$; majority brown (82.65\%); single $(46.47 \%) ; 70.71 \%$ of births were at term; there was a predominance of vaginal deliveries $(68.98 \%)$, with $30.34 \%$ of cesarean sections; $65.36 \%$ of mothers attended less than seven prenatal consultations; $7.49 \%$ of unborn children with low birth weight; there was a positive correlation between cesarean deliveries and: age groups 25 to 35 years $(r=0.974 ; p<0.001)$; mothers over 35 years old $(r=0.836 ; p=0.019)$; education between 8 and 11 years $(r=0.936 ; p=0.012)$; multiple pregnancies $(r=0.652 ; p=0.024)$; low birth weight $(r=0.635 ; p=0.048)$. Conclusion: The SINASC data allowed to characterize the maternal and child population in this micro-region, despite the large number of ignored information, reinforcing the need for training and greater attention to completing the DNV, in order to generate information capable of contributing to the planning of actions aimed at this group.
\end{abstract}

Key words: Live births, Epidemiological profile, Maternal health, Public health.

\footnotetext{
${ }^{1}$ Universidade Federal do Maranhão, Pinheiro - MA. * E-mail: deyvison0010.bol@gmail.com

2 Universidade Federal do Maranhão, São Luís - MA.
} 


\section{RESUMEN}

Objetivo: Caracterizar y analizar el perfil epidemiológico de los nacimientos en Baixada Maranhense, en el período 2011-2017. Métodos: Estudio epidemiológico descriptivo, retrospectivo, con enfoque cuantitativo, utilizando datos de Declaraciones de Nacidos Vivos (DNV), disponibles en el sitio web del SINASC. Resultados: El número total de nacidos vivos fue de 66.972 , siendo el $51,5 \%$ varones; $91,8 \%$ ocurrió en un ambiente hospitalario; madres de 10 a 24 años (61,76\%); mayoría marrón (82,65\%); soltero (46,47\%); El $70,71 \%$ de los nacimientos fueron a término; hubo predominio de partos vaginales $(68,98 \%)$, con $30,34 \%$ de cesáreas; $65,36 \%$ de las madres asistieron a menos de siete consultas prenatales; $7,49 \%$ de los niños por nacer con bajo peso al nacer; hubo una correlación positiva entre los partos por cesárea y: grupos de edad de 25 a 35 años $(r=0,974 ; p<0,001)$; madres mayores de 35 años $(r=0,836 ; p=0,019)$; educación entre 8 y 11 años $(r=0,936 ; p=0,012)$; embarazos múltiples $(r=0,652 ; p=0,024)$; bajo peso al nacer $(r=0,635 ; p$ $=0,048)$. Conclusión: Los datos del SINASC permitieron caracterizar a la población maternoinfantil de esta microrregión, a pesar de la gran cantidad de información ignorada, reforzando la necesidad de capacitación y mayor atención a la cumplimentación de la DNV, con el fin de generar información capaz de contribuir a la planificación de acciones dirigidas a este colectivo.

Palabras clave: Nacidos vivos, Perfil epidemiológico, Salud materna, Salud pública.

\section{INTRODUÇÃO}

Parto e nascimento são eventos transformadores de vida e, na maioria das vezes, não representam riscos para mulher e criança. A gestação, apesar de ser um processo fisiológico, gera mudanças de ordem física, psicológica e social na mulher (LEAL MC, et al., 2014). Muitas dessas alterações são associadas a fatores de natureza biológica, aspectos socioeconômicos e culturais, carecendo de acompanhamento qualificado por equipes multiprofissionais de saúde para que intercorrências sejam evitadas e prevenidas (PEIXOTO CR, et al., 2012).

Políticas efetivas nessa temática são indispensáveis para gestão em saúde e demandam alimentação de informações sobre nascidos vivos para o planejamento e assistência ao parto e ao nascituro (PEDRAZA DF, 2012). Além disto, favorecem a construção de indicadores de saúde e demográficos, tais como a taxa de mortalidade infantil, neonatal e perinatal e as taxas de fecundidade e natalidade (DATASUS, 2012). Nesse sentido, o Ministério da Saúde brasileiro vem implantando diversos Sistemas de Informação (SIS) em Saúde para melhor catalogação e gestão dessas informações. Os SIS são ferramentas importantes para conhecer a realidade, pois os dados produzidos por eles, além de permitir análises epidemiológicas, subsidiam também decisões para a construção de política, planejamento, monitoramento e avaliação de programas de saúde (JESUS A et al, 2018).

Em 1998, foi criado o Sistema de Informações sobre Nascidos Vivos (SINASC), representando um avanço na forma de coletar e processar dados demográficos e epidemiológicos desde o pré-natal até o parto (SILVA MB, 2018; DATASUS, 2020). Esse sistema é alimentado por meio da informatização das Declarações de Nascidos Vivos (DNV), emitidas pela Secretaria de Vigilância em Saúde e distribuídas pelas Secretariais Estaduais de Saúde às Secretarias Municipais de Saúde; fato que permite obter informações epidemiológicas dos nascidos de acordo com as seguintes variáveis: sexo, peso ao nascer, idade gestacional, duração da gestação, tipo de parto, idade da mãe, entre outras (DATASUS, 2020). Outrora, os registros de nascimentos estavam baseados exclusivamente no Sistema de Registro Civil, que compreendia apenas nascimentos informados em cartórios, apresentando níveis significativos de sub-registro, não refletindo a realidade (DE ALMEIDA MF, et al., 2009).

A emissão da DNV é obrigatória em estabelecimentos e serviços de saúde que realizam partos, e o preenchimento de suas informações deve ser realizado pelos profissionais que prestaram assistência ao parto ou ao recém-nascido, sendo obrigatória também para cartórios de Registro Civil até nos casos de partos domiciliares sem assistência. Quando inseridos no SINASC, os dados da DNV tornam-se disponíveis para a 
população por meio da página do DATASUS na internet, com atualização das publicações a cada dois anos (ANDRADE SM, et al., 2017).

Por meio dessas informações é possível conhecer o perfil epidemiológicos dos nascidos vivos e das parturientes, bem como as condições referentes à assistência em saúde do binômio mãe-feto, uma vez que - SINASC oferece um banco de dados simplificado e fidedigno, sendo indispensável para gestores da saúde, por direcionar políticas públicas e dar subsídios para o planejamento de ações de saúde relacionadas à mulher e à criança, em todos os níveis de atenção (ANDRADE SM, et al., 2017).

Diante disso, a realização do presente estudo teve como objetivo caracterizar e analisar o perfil epidemiológico dos nascidos vivos e das parturientes da região da Baixada Maranhense, no estado do Maranhão; além de trazer à comunidade acadêmica dados catalogados que facilitem a discussão sobre a temática.

\section{MÉTODOS}

Trata-se de um estudo epidemiológico descritivo, retrospectivo, de abordagem quantitativa, baseado em dados que compõem o perfil epidemiológico dos nascimentos da Baixada Maranhense - microrregião definida pelo Instituto Brasileiro de Geografia e Estatística (IBGE). A fonte dos dados utilizados foram as DVN's, por meio da base secundária do SINASC disponíveis no Departamento de Informática do Sistema Único de Saúde (DATASUS), compreendendo o período entre 2011 e 2017.

As variáveis analisadas a partir das DVN's foram: idade, estado civil, raça cor, escolaridade, consultas de pré-natal, tipo de gravidez, idade gestacional, tipo de parto, sexo, peso ao nascer, Apgar no primeiro minuto, Apgar no quinto minuto e presença de anomalia congênita.

Os dados foram tabulados e organizados no Microsoft Office Exce ${ }^{\circledR}$ 2016, depois da importação do DATASUS. A análise dos dados foi realizada com o software IBM ${ }^{\circledast}$ SPSS ${ }^{\circledast}$ Statistics 20 (2011). As variáveis que tiveram distribuição normal, o teste de correlação de Pearson foi empregado, e para aquelas com distribuição não normal, o teste de correlação de Spearman's rank. Para o grau de associação entre as variáveis analisadas e suas relações se admitiu a classificação de Dancey C e Reidy J (2006). Em todos os testes, o nível de significância ( $\alpha$ ) utilizado foi de $5 \%$, ou seja, foi considerado significativo quando $p<0,05$.

Por envolver apenas dados secundários e de domínio público, este estudo não necessitou de aprovação por parte do Comitê de Ética em Pesquisa, fundamentando-se nas diretrizes e normas regulamentadoras estabelecidas nas Resoluções nº 466/2012 e 580/2018 do Conselho Nacional de Saúde do Ministério da Saúde.

\section{RESULTADOS E DISCUSSÃO}

Os dados do SINASC, no período entre 2011 e 2017, registram 66.972 nascidos vivos na Baixada Maranhense, apresentando como média anual aproximadamente 9.568 nascimentos, sendo $51,50 \%$ do total categorizados como do sexo masculino. Do período avaliado, os anos 2011 e 2016, respectivamente, apresentaram o maior e o menor número de nascimentos, correspondendo a $10.116(15,10 \%)$ e 9.216 $(13,76 \%)$.

Foi possível observar que existe uma tendência linear de decréscimo na Baixada Maranhense do número de nascimentos, conforme o Gráfico 1. Esses dados divergem de achados encontrados por Silva MB (2018) no Pernambuco, também localizado na região nordeste, que demonstraram crescimento de nascimentos dentro do período avaliado; fato que, talvez, demonstre estratégias diferentes de planejamento familiar abordadas pelas agências de saúde locais e também por questões socioeconômicas da polução avaliada. 
Gráfico 1 - Número de nascidos vivos e sexo na Baixada Maranhense no período de 2011 a 2017.

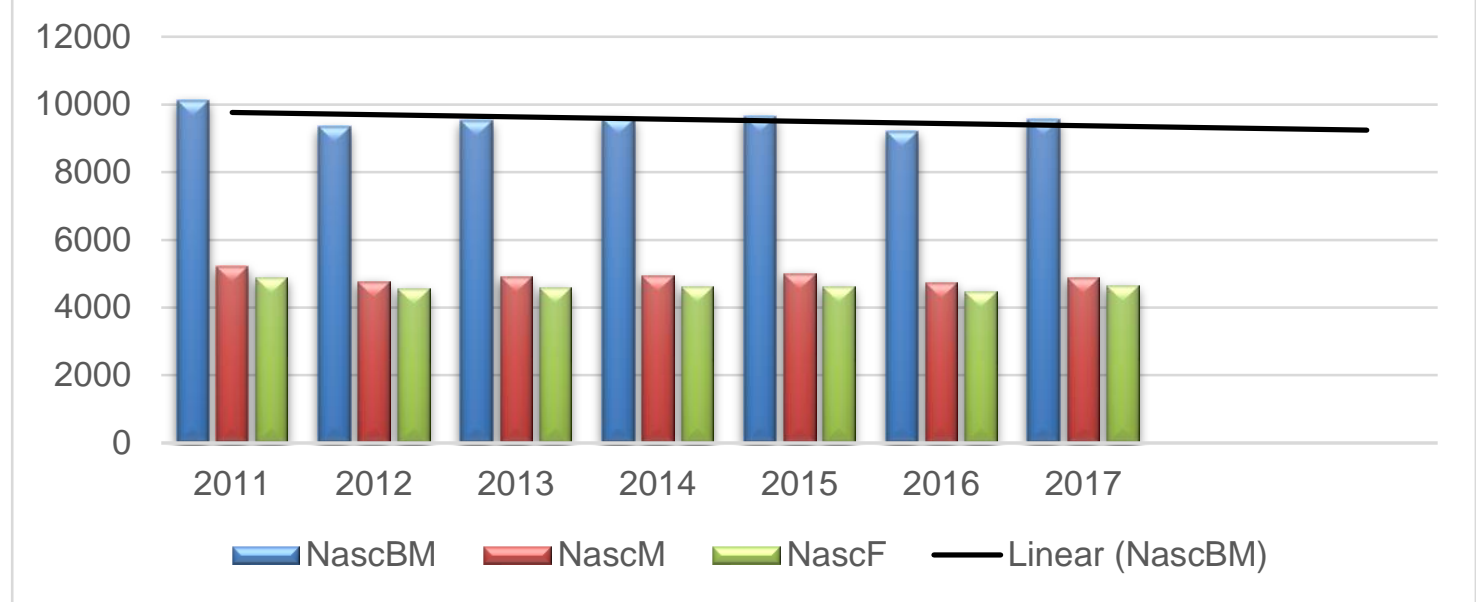

Legenda: NascBM = nascidos vivos na Baixada Maranhense; $\mathrm{NascM}=$ nascidos vivos do sexo masculino; NascF = nascidos vivos do sexo feminino.

Fonte: Lopes DL, et al., 2021, dados extraídos de SINASC/ DATASUS/MS.

Em relação ao local de parto dos nascidos vivos, a Tabela 1 evidencia que $61.492(91,81 \%)$ dos conceptos nasceram no ambiente hospitalar e apenas 4.549 (6,79\%) em domicílio, corroborando com outros estudos que também atestaram predominância de nascimentos em ambientes hospitalares (CORREIO RAS, et al., 2016; SILVA MB, 2018). Silva ZP (2008) e Silva EAT (2013) destacam que a crescente urbanização, as mudanças de hábitos culturais e a expansão dos serviços de saúde favoreceram a institucionalização do parto que outrora era assistido em domicílio, sendo essa mudança um fator de contribuição para uma assistência mais ampla e menor risco possível ao binômio mãe-feto.

Tabela 1 - Local de ocorrência do nascimento dos nascidos vivos na Baixada Maranhense, no período de 2011 a 2017.

\begin{tabular}{ccc}
\hline Local do Nascimento & Número de Nascidos Vivos & $\%$ \\
\hline Hospital & 61.492 & 91,81 \\
Outro estabelecimento de Saúde & 847 & 1,26 \\
Domicílio & 4549 & 6,79 \\
Outro & 80 & 0,11 \\
Ignorado & 4 & 0,03 \\
\hline Total & $\mathbf{6 6 . 9 7 2}$ & $\mathbf{1 0 0}$
\end{tabular}

Fonte: Lopes DL et al. (2021), dados extraídos de SINASC/ DATASUS/MS.

O perfil das características maternas pode ser observado na Tabela 2. Constata-se uma maior prevalência de puérperas nas faixas de 10 a 24 anos e uma menor na faixa igual ou superior aos 35 anos, corroborando com trabalhos nacionais que também identificaram perfil semelhante de faixas etárias (CORREIO RAS, et al., 2016; SANTOS GM, et al., 2017; SILVA MB, 2018). Azevedo WF, et al. (2015) e Takagi MM, et al. (2018) destacam que o desenvolvimento de uma gestação está diretamente correlacionado com a faixa etária da mulher, sendo a mais precoce e a mais tardia um fator de risco para possíveis complicações com a mulher e com a criança.

Azevedo WF, et al. (2015) também descreve preocupação com gravidez em estrato etário que envolve a adolescência e afirma que esse fenômeno não é recente, apresentando, ainda, significativa representação nos índices nacionais e, principalmente regionais; fato que repercute em problemas sociais e de saúde pública, pois traz consequências para vida inteira da genitora devido principalmente à alteração no seu desenvolvimento psicossocial. Por outro lado, em virtude da crescente profissionalização da mulher, estabilização financeira e dinâmicas de planejamento familiar, a literatura tem demostrado uma tendência maior de gravidez na faixa etária superior aos 35 anos (CORREIO RAS, et al., 2016; SANTOS GM, et al., 2017). 
Tabela 2 - Perfil das características sociodemográficas e dados obstétricos na Baixada Maranhense no período de 2011 a 2017.

\begin{tabular}{|c|c|c|}
\hline Variável & $\mathbf{N}$ & $\%$ \\
\hline \multicolumn{3}{|l|}{ Idade } \\
\hline $10-24$ & 41.362 & 61,760 \\
\hline $25-34$ & 22.000 & 32,850 \\
\hline$\geq 35$ & 3.608 & 5,387 \\
\hline Ignorado & 2 & 0,003 \\
\hline Total & 66.972 & 100 \\
\hline \multicolumn{3}{|l|}{ Raça/cor } \\
\hline Branca & 5.657 & 8,447 \\
\hline Preta & 4.030 & 6,017 \\
\hline Amarela & 97 & 0,145 \\
\hline Parda & 55.351 & 82,648 \\
\hline Indígena & 75 & 0,112 \\
\hline Ignorado & 1.762 & 2,631 \\
\hline Total & 66.972 & 100 \\
\hline \multicolumn{3}{|l|}{ Estado Civil } \\
\hline Solteira & 31118 & 46,464 \\
\hline Casada & 6391 & 9,543 \\
\hline Viúva & 129 & 0,193 \\
\hline Separada Judicialmente & 153 & 0,228 \\
\hline União Estável & 27967 & 41,759 \\
\hline Ignorado & 1214 & 1,813 \\
\hline Total & 66.972 & 100 \\
\hline \multicolumn{3}{|l|}{ Escolaridade } \\
\hline Nenhuma & 821 & 1,226 \\
\hline$\leq 8$ anos & 22.754 & 33,975 \\
\hline$>8$ e $\leq 11$ anos & 39.210 & 58,547 \\
\hline$\geq 12$ anos & 2919 & 4,359 \\
\hline Ignorado & 1268 & 1,893 \\
\hline Total & 66.972 & 100 \\
\hline \multicolumn{3}{|c|}{ Duração da gestação (semanas) } \\
\hline$<37$ & 8434 & 12,593 \\
\hline $37-41$ & 47356 & 70,710 \\
\hline$\geq 42$ & 5443 & 8,127 \\
\hline Ignorado & 5739 & 8,569 \\
\hline Total & 66.972 & 100 \\
\hline \multicolumn{3}{|l|}{ Tipo de parto } \\
\hline Vaginal & 46198 & 68,981 \\
\hline Cesáreo & 20323 & 30,346 \\
\hline Ignorado & 451 & 0,673 \\
\hline Total & 66.972 & 100 \\
\hline \multicolumn{3}{|l|}{ Tipo de gravidez } \\
\hline Única & 65675 & 98,063 \\
\hline Dupla & 928 & 1,386 \\
\hline Tripla & 28 & 0,042 \\
\hline Ignorado & 341 & 0,509 \\
\hline Total & 66.972 & 100 \\
\hline \multicolumn{3}{|c|}{ Número de consultas pré-natal } \\
\hline Nenhuma & 4470 & 6,674 \\
\hline$<7$ consultas & 43.777 & 65,366 \\
\hline$>7$ consultas & 18.349 & 27,398 \\
\hline lgnorado & 376 & 0,561 \\
\hline Total & 66.972 & 100 \\
\hline
\end{tabular}

Fonte: Lopes DL, et al., 2021, dados extraídos de SINASC/ DATASUS/MS. 
Para os dados relacionados à raça/cor das puérperas, evidenciou-se que a cor parda foi a que mais prevaleceu (82,64\%), corroborando com outro estudo realizado na mesma região (Tabela 2) (SILVA MB, 2018). Esse resultado deve ser interpretado a partir dos dados demográficos disponíveis no último censo demográfico do IBGE, no qual a região Nordeste apresentou $69,22 \%$ de população autodeclarada parda e ou negra, seguidas de $29,2 \%$ de pessoas autodeclaradas brancas (IBGE, 2020).

No que concerne ao estado civil das puérperas, os dados obtidos revelam um predomínio de mães solteiras. Resultado este que se contrapõe ao descrito por Silva MB (2018) ao avaliar o perfil epidemiológico das puérperas e dos recém-nascidos no Estado do Piauí, observando prevalência de mães casadas. Essa constatação na Baixada Maranhense é preocupante, visto que a situação conjugal das puérperas é relevante e influenciadora para um aporte psicológico do cônjuge que o momento impõe; além de se materializar como um determinante de risco para não adesão ao pré-natal (SILVA FCB, 2009).

Segundo o Caderno de Atenção Básica ao Pré-Natal de Baixo Risco, elaborado pelo Ministério da Saúde, a baixa escolaridade materna também implica diretamente na utilização dos serviços de saúde, como na realização do pré-natal, sendo que neste levantamento foi evidenciado que $58,54 \%$ das mães de nascidos vivos possuem entre 8 a 11 anos de estudo; fato que se contrasta com outros dados da região Nordeste e Centro-Oeste (BRASIL, 2012; FERREIRA VA, et al., 2018; SANTOS GM, et al., 2017). No entanto, a preocupação se apresenta maior ao observarmos o número de mulheres com baixa escolaridade na microrregião avaliada, com porcentagem inferior ao relatado em trabalhos de cunho semelhante a nível nacional (SANTOS GM, et al., 2017; SILVA MB, 2018).

Constatou-se, também, que a duração da gestação mais prevalente foi a termo, sendo este um achado semelhante ao encontrado por Silva MB (2018). Contudo, o percentual de nascidos vivos abaixo de 37 semanas $(12,59 \%)$ catalogados demonstra valores superiores ao já descrito na literatura para outras regiões (SANTOS GM, et al., 2017). A duração da gestação é relevante para as análises de perfil de partos porque se relaciona ao componente de mortalidade infantil no período neonatal e aumenta o risco de morbidades nos neonatos, acarretando uma necessidade de assistência com maior complexidade e aumento de custos para o Sistema Único de Saúde (SUS) (BALBI B, et al., 2016).

O Ministério da Saúde recomenda, como ideal, o número mínimo de seis consultas pré-natal (BRASIL, 2012). A prevalência de puérperas que realizaram menos de sete consultas está em torno de $72,03 \%$ das ocorrências, conforme Tabela 2. Domingues RMSM, et al. (2015) destacam que a menor adequação do prénatal é observada em mulheres mais jovens, de pele preta, multíparas, sem companheiro, sem trabalho remunerado, com menos anos de estudo, de classes econômicas mais baixas e residentes nas regiões Norte e Nordeste do país, reiterando o achado previamente descrito do quantitativo de gestantes com de baixa escolaridade e idade.

Em relação ao tipo de gravidez e parto, identificou-se maior ocorrência de gravidez com feto único $(98,06 \%)$ e parto por via vaginal $(68,98 \%)$, corroborando com outros trabalhos nacionais já (CORREIO RAS, et al., 2016; SANTOS GM, et al., 2017). Nomura RMY, et al. (2004) evidenciou em um hospital universitário taxa menor de complicações materno e neonatais quando o parto ocorria por via vaginal, levando a acreditar que a predominância desses partos na Baixada Maranhense configura uma proteção aos nascituros.

A predominância de nascimentos do sexo masculino sobre o feminino constatado corrobora com diversas pesquisas epidemiológicas sobre recém-nascidos em outros estados brasileiros (Tabela 3) (CORREIO RAS, et al., 2016; SANTOS GM, et al., 2017; SILVA MB, 2018; FERREIRA VA, et al., 2018).

Em relação ao peso dos nascituros, o percentual abaixo de $2500 \mathrm{~g}$ englobou 7,49\% dos nascidos, e apesar desse valor ser encontrado também noutros trabalhos, observa-se que em países desenvolvidos essa porcentagem é inferior a 6\% (UCHUMURA TT, et al., 2008; SILVA MB, 2018; MOREIRA AIM, et al., 2018; ABRINQ, 2019). 
Tabela 3 - Perfil das características perinatais dos nascidos vivos na Baixada Maranhense no período de 2011 a 2017.

\begin{tabular}{|c|c|c|}
\hline Variável & $\mathbf{N}$ & $\%$ \\
\hline \multicolumn{3}{|l|}{ Sexo } \\
\hline Masculino & 34.488 & 51,49 \\
\hline Feminino & 32.468 & 48,47 \\
\hline Ignorado & 16 & 0,02 \\
\hline Total & 66.972 & 100 \\
\hline \multicolumn{3}{|c|}{ Peso ao nascer (gramas) } \\
\hline$<1500$ & 675 & 1,01 \\
\hline 1500 a 2499 & 4.343 & 6,48 \\
\hline 2500 a 3999 & 14.423 & 21,53 \\
\hline$>3999$ & 47.393 & 70,76 \\
\hline Ignorado & 138 & 0,20 \\
\hline Total & 66.972 & 100 \\
\hline \multicolumn{3}{|c|}{ Apgar no primeiro minuto } \\
\hline$<5$ & 3.062 & 4,57 \\
\hline 6 a 7 & 7.823 & 11,68 \\
\hline 8 a 10 & 39.758 & 59,36 \\
\hline Ignorado & 16.329 & 24,38 \\
\hline Total & 66.972 & 100 \\
\hline \multicolumn{3}{|c|}{ Apgar no quinto minuto } \\
\hline$<5$ & 688 & 1,02 \\
\hline 6 a 7 & 1.816 & 2,71 \\
\hline 8 a 10 & 47.448 & 70,84 \\
\hline Ignorado & 17.020 & 25,41 \\
\hline Total & 66.972 & 100 \\
\hline \multicolumn{3}{|c|}{ Anomalia congênita } \\
\hline Sim & 163 & 0,24 \\
\hline Não & 64.199 & 95,85 \\
\hline Ignorado & 2.610 & 3,89 \\
\hline Total & 66.972 & 100 \\
\hline
\end{tabular}

Fonte: Lopes DL, et al., 2021, dados extraídos de SINASC/ DATASUS/MS.

O peso ao nascer repercute na vida e sobrevida do neonato, bem como no crescimento e desenvolvimento da criança, além de influenciar na morbidade, mortalidade e favorecer o aparecimento de doenças de risco em idade adulta (PEDRAZA DF, 2012; LIMA MCBM, et al., 2013).

A categoria de peso entre 3.000 gramas e 3.999 gramas é considerada a mais favorável, por representar um fator de proteção à mortalidade infantil. Os números da mortalidade materna e neonatal estão relacionados à falta de reconhecimento deste problema como sendo social e político, além do desconhecimento da sua magnitude e da deficiência na qualidade dos serviços de saúde no Brasil (LIMA MCBM, et al., 2013)

O boletim de Apgar no 1ํ e 5ํำ minuto, que utiliza critérios como frequência cardíaca, esforço respiratório, tônus muscular, irritabilidade reflexa e cor, é um importante indicador de risco para a morbimortalidade neonatal, pois permite descrever condições do recém-nascido, atribuindo pontuação de 0 a 20 minutos (BRASIL, 2012). Os achados deste estudo foram diferentes dos descritos por Silva MB (2018) e Ferreira VA, et al. (2018) no qual os índices representaram cerca de $85 \%$ no primeiro e quinto minuto para pontuação de 8 a 10 (Tabela 3).

Não vencida a questão epidemiológica, fez-se necessário o cruzamento de informações do tipo de parto e fatores correlacionados (Tabela 4). Nos últimos anos, por exemplo, existe um paradoxo do nascimento frente a medicalização e/ou intervenções médicas, onde a hipermedicalização e uso desnecessário de tecnologias em saúde na assistência à gestação representam também riscos às mulheres e recém-nascidos; sendo a cesárea uma dessas tecnologias (SOUZA JP e PILEGGI-CASTRO C, 2014). 
Tabela 4 - Correlação das variáveis relacionadas ao tipo de parto com as variáveis puerperais, obstétricas e dos recém-nascidos na Baixada Maranhense no período de 2011 a 2017.

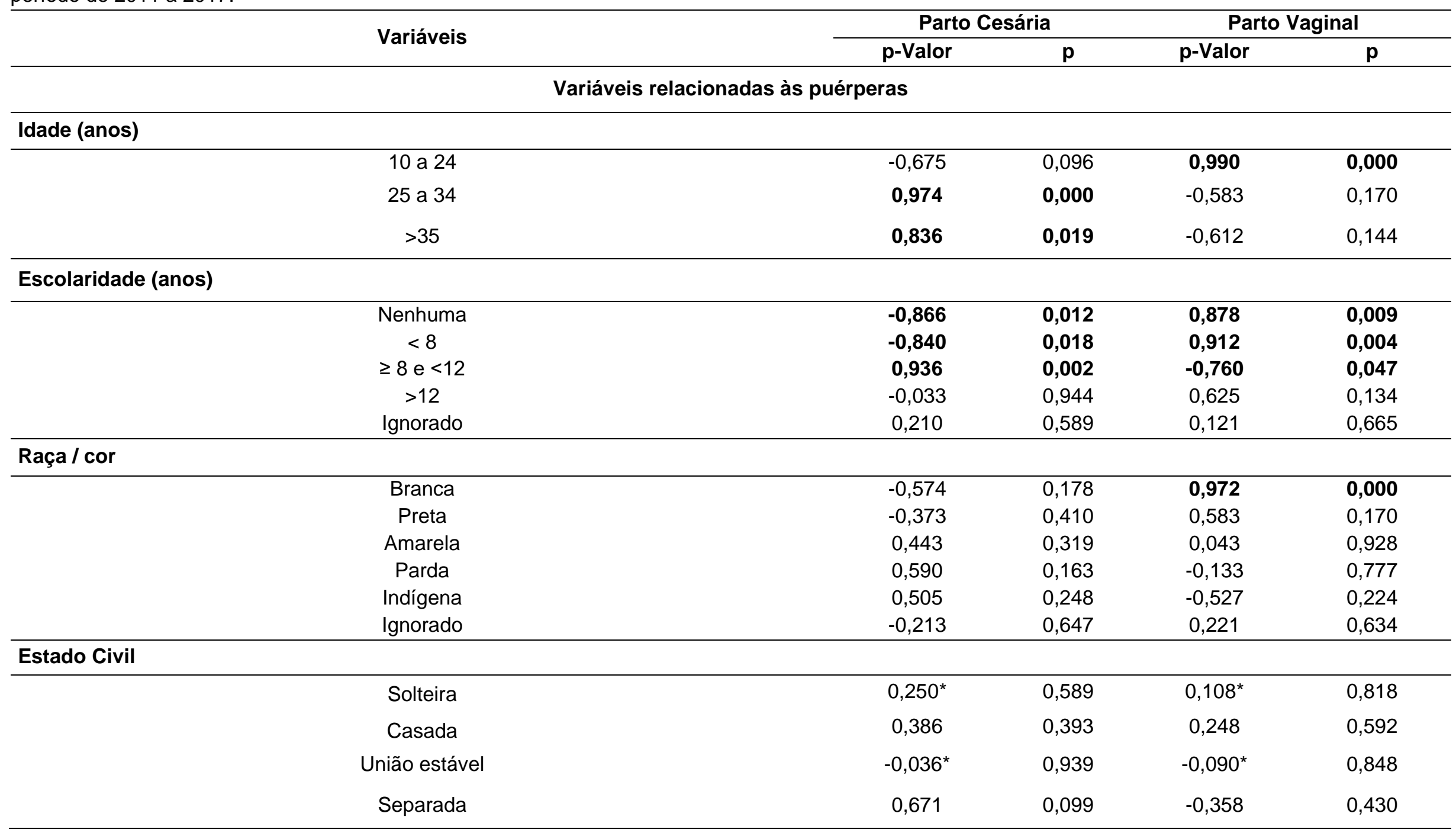




\begin{tabular}{|c|c|c|c|c|}
\hline \multirow{2}{*}{ Variáveis } & \multicolumn{2}{|c|}{ Parto Cesária } & \multicolumn{2}{|c|}{ Parto Vaginal } \\
\hline & p-Valor & $\mathbf{p}$ & p-Valor & $\mathbf{p}$ \\
\hline \multicolumn{5}{|c|}{ Variáveis relacionadas à gestação e ao parto } \\
\hline \multicolumn{5}{|l|}{ Número de consultas pré-Natal } \\
\hline Nenhuma & $-0,827$ & 0,022 & 0,539 & 0,212 \\
\hline$<7$ & $-0,271$ & 0,556 & 0,756 & 0,049 \\
\hline$\geq 7$ & 0,665 & 0,103 & $-0,548$ & 0,203 \\
\hline Ignorado & $-0,293$ & 0,523 & 0,778 & 0,039 \\
\hline \multicolumn{5}{|l|}{ Idade Gestacional (semanas) } \\
\hline$<37$ semanas & 0,496 & 0,258 & $-0,614$ & 0,143 \\
\hline De 37 a 41 semanas & 0,236 & 0,610 & 0,397 & 0,377 \\
\hline$\geq 42$ semanas & $-0,376$ & 0,405 & 0,674 & 0,097 \\
\hline Ignorado & $-0,758$ & 0,048 & 0,704 & 0,077 \\
\hline \multicolumn{5}{|l|}{ Tipo de gravidez } \\
\hline Única & $-0,722$ & 0,068 & 0,226 & 0,625 \\
\hline Múltipla & 0,652 & 0,024 & $-0,587$ & 0,042 \\
\hline Ignorado & $-0,72$ & 0,068 & 0,392 & 0,385 \\
\hline \multicolumn{5}{|l|}{ Local de Nascimento } \\
\hline Hospital & 0,575 & 0,045 & 0,203 & 0,663 \\
\hline Domicílio & $-0,840$ & 0,018 & 0,943 & 0,001 \\
\hline Outros & $-0,410$ & 0,931 & 0,215 & 0,644 \\
\hline Ignorado & 0,246 & 0,358 & 0,421 & 0,586 \\
\hline
\end{tabular}




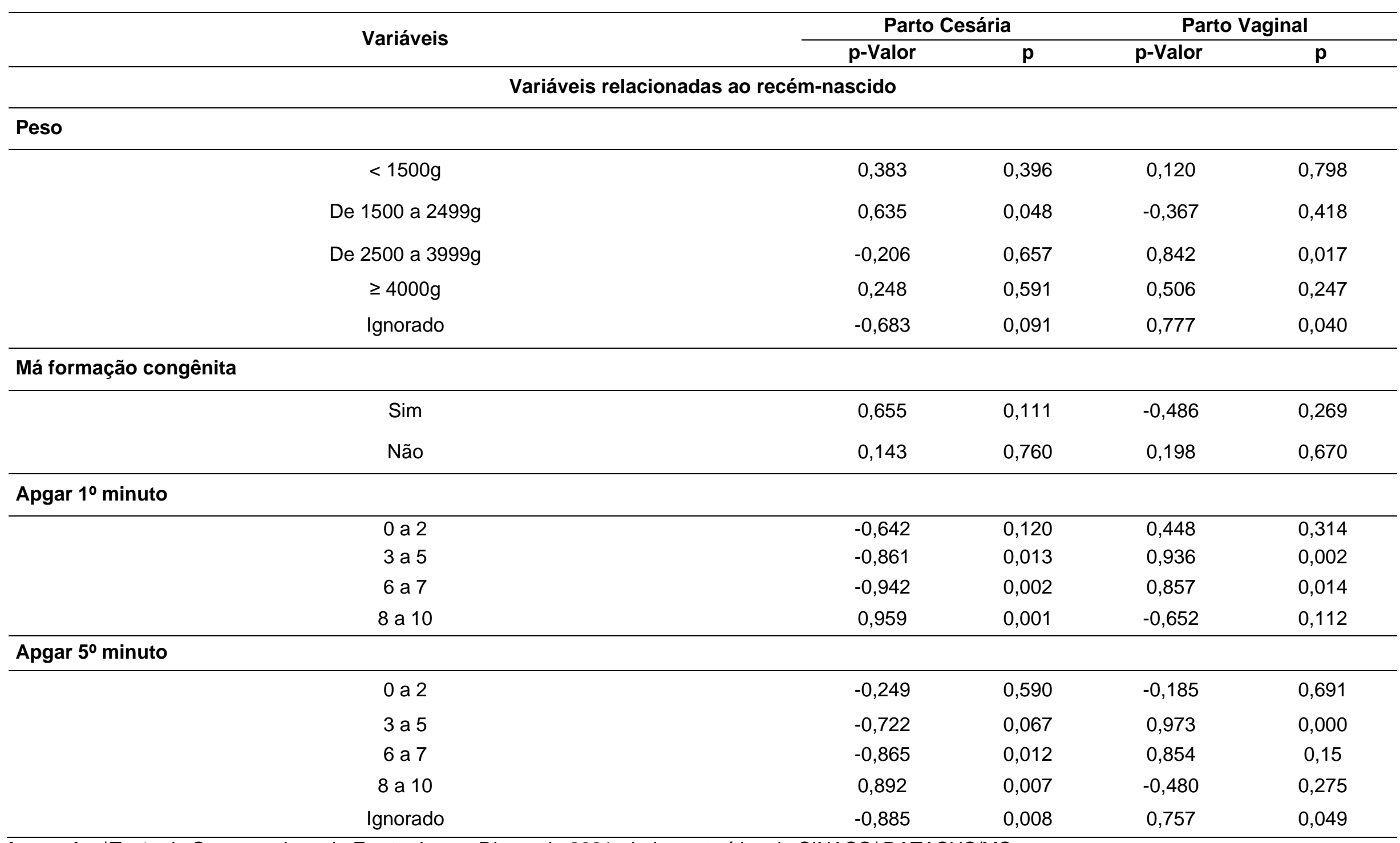

Legenda: *Teste de Spearman's rank. Fonte: Lopes DL, et al., 2021, dados extraídos de SINASC/ DATASUS/MS. 
No Estado do Maranhão, de acordo com o SINASC, de 2011 a 2017 ocorreram 810.261 partos (DATASUS, 2020). Desse total, mais de $41,1 \%$ foram por meio de cesarianas, ou seja, cerca de $10 \%$ maior do que as taxas encontradas na Baixada para o mesmo intervalo de tempo (DATASUS, 2020). Segundo a Organização Mundial de Saúde (OMS), o índice razoável de cesáreas é de 10\% a 15\% dos nascimentos, não havendo justificativa para qualquer região do mundo ter taxas maiores (OMS, 2015).

Estudos realizados no Brasil destacam também a influência de aspectos socioculturais, tais como idade, escolaridade e etnia na prevalência dessa ocorrência, além da associação dessa prática com baixo peso ao nascer e o nascimento pré-termo (SAKAE TM, et al., 2009; PADUA KS, et al., 2010; LEAL MC, et al., 2017). Essas evidências corroboram com dados obtidos na correlação entre as variáveis parto cesáreo e as características maternas e dos recém-nascidos, em nosso estudo (Tabela 4).

Diante dos dados, não se evidenciou achados significativos sobre a raça/cor e a via de parto. Leal MC, et al. (2017), diferentemente dos nossos resultados, apontaram achados que remetem risco de morte de puérperas de cor preta e parda devido a um pré-natal inadequado, além de ausência de acompanhantes, peregrinação para o parto e menos anestesia para a episiotomia em comparação às brancas.

Partos por via vaginal se correlacionam à faixa etária de 10 a 24 anos, enquanto que as cesarianas estão correlacionadas positivamente com a faixa etária entre 25 e 34 anos e em gestantes maiores que 35 anos. Estes dados corroboram com Padua KS, et al. (2010), principalmente na faixa etária maior que 35 anos. Esse fato pode ter relação com as pacientes estarem mais sujeitas a intercorrências clínicas, além de uma monitorização médica mais próxima delas, sendo consideradas pelos médicos, em sua maioria, mais seguro submetê-las ao parto cesáreo (PADUA KS, et al., 2010).

A escolaridade é um fator que influencia no tipo de parto, sendo observado correlação negativa entre puérperas que tinham menos que 8 anos de estudo e positiva naquelas que apresentavam escolaridade entre 8 e 11 anos à cesariana. Freitas PF, et al. (2008) encontraram correlação semelhante e discorrem sob a premissa que a baixa escolaridade pode indicar mudanças de atitude do sistema público em promover o parto vaginal como preferencial; teoria que ainda não se efetivou. Rattner D, et al. (2016) e Guimães RM, et al. (2017) contrapõem essa ideia e descrevem que o maior acesso à educação leva a mulher a escolher o parto cesáreo na crença de sofrerem menos e preservarem o canal vaginal.

Diferentemente de estudos (que evidenciam que o maior número de consultas pré-natal está relacionado a via de parto, nossa pesquisa não encontrou uma correlação estatisticamente significativa com essa premissa (VALOR) (PADUA KS, et al., 2010; DOMINGUES RMSM, et al., 2015). No entanto, mostrou que houve correlação estatisticamente negativa com a realização de cesarianas e o número de consultas menores que sete, ou seja, quanto mais gestantes com número de consultas inferior ao preconizado pelo Ministério da Saúde, menores as chances de parto cesáreo.

Observou-se, também, que o número de crianças com Baixo Peso ao Nascer (BPN) esteve positivamente correlacionada com partos por via cesariana $(r=0,635 ; p=0,048)$. Leal MC, et al. (2014) e Leal MC, et al. (2017), descrevem risco de ocorrência de BPN em partos do tipo cesáreo; mas dessa relação, a associação se apresenta de maneira complexa na realidade, visto que o procedimento pode ser indicado nas condições clínicas ou obstétricas relacionadas com complicações para a gestação ou para o feto, e as indicações, por si só, já representam um risco para o BPN.

Cesarianas levam ao aumento da morbimortalidade materna e neonatal, nascimento pré-termo, infecção puerperal e o BPN; além de associarem a retardo na recuperação puerperal, maior tempo de internação, maior uso de medicamentos, início tardio da amamentação e, por fim, elevação de gastos para o sistema de saúde. $O$ incremento deste procedimento não se deve apenas a questões médicas, sendo influenciado por diversos outros fatores relacionados à gestante, como as desigualdades socioeconômicas e a faixa etária (PADUA KS, et al., 2010; SANCHES NC, et al., 2012; SANTOS GM, et al., 2017; LEAL MC, et al., 2017). Essa constatação torna-se um alerta para uma maior investigação dessa problemática na microrregião da Baixada Maranhense afim de que possa prevalecer a prática médica baseada em evidências e não em questões econômico-sociais. 


\section{CONCLUSÃO}

Diante dos dados correlacionados, foi possível observar o perfil de partos e nascimentos na Baixada Maranhense e lançar bases para discussão, principalmente no quesito setorial/regional e de outros locais com as mesmas características pelo Brasil para efetivação de atividades específicas ao público, melhorando a qualidade de vida da população e diminuindo custos desnecessários ao SUS. Os dados aportados no Sistema de Informação em Saúde, especialmente o SINASC, são uma excelente ferramenta para geração de relatórios que favoreçam essa individualidade, sendo sua manutenção e expansão, por meio de gestão em saúde, um mecanismo de melhoria continuada do SUS.

\section{REFERÊNCIAS}

1. ALMEIDA MF, et al. Sistema de Informações sobre nascidos Vivos-Sinasc: uma avaliação de sua trajetória. A experiência brasileira em sistemas de informação em saúde, p. 11, 2009.

2. ANDRADE SM, et al. Epidemiologia e Indicadores de Saúde. In: ANDRADE SM, et al. (Org.), Bases da saúde coletiva, 2. ed. Paraná: Eduel. 2017. cap. 10, p. 183-210.

3. AZEVEDO WF, et al. Complications in adolescent pregnancy: systematic review of the literature. Einstein (São Paulo), $2015 ; 13(4): 618-626$.

4. BALBI B, et al. Tendência temporal do nascimento pré-termo e de seus determinantes em uma década. Ciência \& Saúde Coletiva, 2016; 21: 233-241.

5. BRASIL. MINISTÉRIO DA SAÚDE. Caderno de atenção básica no 32: atenção ao pré-natal de baixo risco. Brasília; $2012 ; 320 \mathrm{p}$.

6. CORREIO RAS, et al. Perfil epidemiológico dos nascidos vivos no município de Chapecó-SC. Revista Eletrônica de Comunicação, Informação e Inovação em Saúde, 2016; 10 (2): 1-16.

7. DANCEY C, REIDY J. Estatística Sem Matemática para Psicologia: Usando SPSS para Windows. Porto Alegre, Artmed, 2006; 608p.

8. DATASUS. 2020. Departamento de Informática do Sistema Único de Saúde do Brasil. Disponível em: www.datasus.gov.br. Acesso em: 23 nov. 2020.

9. DOMINGUES RMSM, et al. Adequacy of prenatal care according to maternal characteristics in Brazil. Revista Panamericana de Salud Publica, 2015; 37(3): 140-148.

10. FERREIRA VA, et al. Perfil epidemiológico dos nascidos vivos em Belo Horizonte, MG, Brasil. Revista de Enfermagem do Centro Oeste Mineiro, 2018; 8: 1-9.

11. FREITAS PF, et al. Fatores médicos e não-médicos associados às taxas de cesariana em um hospital universitário no Sul do Brasil. Caderno de Saúde Pública, 2008; 24(5): 1051-1061.

12. GUIMARAES RM, et al. Fatores associados ao tipo de parto em hospitais públicos e privados no Brasil. Revista Brasileira de Saúde Materna e Infantil, 2017; 17(3): 571-580.

13. IBGE. 2020. Instituto Brasileiro de Geografia e Estatística. Disponível em: www.ibge.gov.br. Acesso em: 23 nov. 2020.

14. JESUS A, et al. Investigação de informação e comunicação: o boletim epidemiológico como dispositivo interdisciplinar de saúde. 2018. Dissertação (Mestrado em Informação e comunicação em saúde). Fundação Osvaldo Cruz. Rio de Janeiro. 2018

15. LEAL MC, et al. A cor da dor: iniquidades raciais na atenção pré-natal e ao parto no Brasil. Caderno de Saúde Pública, 2017; 33(1).

16. LEAL MC, et al. Determinantes do óbito infantil no Vale do Jequitinhonha e nas regiões Norte e Nordeste do Brasil. Rev. Saúde Pública, 2017; 51.

17. LEAL MC, et al. Sumário Executivo Temático da Pesquisa "Nascer no Brasil". Inquérito Nacional sobre o Parto e Nascimento. Ensp, 2014; 1-8.

18. LIMA MCBM, et al. A desigualdade espacial do Baixo Peso ao Nascer no Brasil. Ciência \& Saúde Coletiva, 2013; 18(8): 2443-2452.

19. MOREIRA AIM, et al. Baixo peso ao nascer e seus fatores associados. Einstein (São Paulo), 2018; 16(4).

20. NOMURA RMY, et al. Complicações maternas associadas ao tipo de parto em hospital universitário. Revista de Saúde Pública, 2004; 38(1): 9-15.

21. ORGANIZAÇÃO MUNDIAL DA SAÚDE (OMS). Declaração da OMS sobre Taxas de Cesáreas. Genebra, 2015.

22. PADUA KS, et al. Fatores associados à realização de cesariana em hospitais brasileiros. Revista de Saúde Pública, 2010; 44(1): 70-79.

23. PEDRAZA DF. Qualidade do Sistema de Informações sobre Nascidos Vivos (SINASC): análise crítica da literatura. Ciência e Saúde Coletiva, 2012; 17: 2729-37. 
24. PEIXOTO CR, et al. Perfil das gestantes atendidas no serviço de pré-natal das unidades básicas de saúde de Fortaleza-CE. Revista Mineira de Enfermagem, 2012; 16(2): 171-177

25. RATTNER D, et al. Nascimentos no Brasil: associação do tipo de parto com variáveis temporais e sociodemográficas. Revista Brasileira de Saúde Materno e Infantil, 2016; 16(1): 39-47.

26. SAKAE TM, et al. Fatores associados a taxas de cesárea no hospital universitário. Revista de Saúde Pública, São Paulo, 2009; 43(3): 472-480.

27. SANCHES NC, et al. Perfil das mulheres submetidas à cesariana e assistência obstétrica na maternidade pública em Ribeirão Preto. Texto \& Contexto Enfermagem, 2012; $21(2)$.

28. SANTOS GM, et al. Análise do perfil das puérperas e dos nascidos vivos em um estado do Nordeste brasileiro. Revista Uningá Review, 2017; 31(1).

29. SILVA EAT. Gestação e preparo para o parto: programas de intervenção. O Mundo da Saúde, São Paulo, 2013; $37(2): 208-215$.

30. SILVA FCB. Experienciando a ausência do companheiro nas consultas de pré-natal. Dissertação (Mestrado em Enfermagem) - Universidade Federal do Rio Grande do Norte. Rio Grande do Norte, 2009; 124 p.

31. SILVA MB. Perfil de Nascimentos de uma região de saúde de Pernambuco, 2011 a 2015. Trabalho de Conclusão de Residência (Especialização em Saúde Coletiva) - Escola de Governo em Saúde Pública de Pernambuco. Pernambuco, 2018; $23 \mathrm{p}$.

32. SILVA ZP. Perfil dos nascimentos e da mortalidade neonatal precoce, segundo local de parto, complexidade hospitalar e rede SUS e não-SUS, região metropolitana de São Paulo. Tese (Doutorado em Epidemiologia) Faculdade de Saúde Pública da Universidade de São Paulo. São Paulo, 2008; 124 p.

33. SOUZA JP, PILEGGI-CASTRO C. Sobre o parto e o nascer: a importância da prevenção quaternária. Caderno de Saúde Pública, 2014; 30: 11-13.

34. TAKAGI MM, et al. Resultados perinatais em gestantes acima de 35 anos. Arquivos Médicos dos Hospitais e da Faculdade de Ciências Médicas da Santa Casa de São Paulo, 2018; 55(3): 108-114.

35. UCHIMURA TT, et al. Baixo peso ao nascer e fatores associados. Revista Gaúcha de Enfermagem, 2008; $29(1): 33$. 